\title{
Steady dark solitary waves emerging from wave-generated meanflow: The role of modulation equations
}

\author{
Thomas J. Bridges ${ }^{\text {a) }}$ \\ Department of Mathematics and Statistics, University of Surrey, Guildford, Surrey GU2 7XH, \\ United Kingdom
}

(Received 31 January 2005; accepted 18 April 2005; published online 21 October 2005)

\begin{abstract}
Various classes of steady and unsteady dark solitary waves (DSWs) are known to exist in modulation equations for water waves in finite depth. However, there is a class of steady DSWS of the full water-wave problem which are missed by the classical modulation equations such as the Hasimoto-Ono, Benney-Roskes, and Davey-Stewartson. These steady DSWs, recently discovered by Bridges and Donaldson, are pervasive in finite depth, arise through secondary criticality of Stokes gravity waves, and are synchronized with the Stokes wave. In this paper, the role of DSWs in modulation equations for water waves is reappraised. The intrinsic unsteady nature of existing modulation equations filters out some interesting solutions. On the other hand, the geometry of DSWs in modulation equations is very similar to the full water wave problem and these geometrical properties are developed. A model equation is proposed which illustrates the general nature of the emergence of steady DSWs due to wave-generated mean flow coupled to a periodic wave. Although the existing modulation equations are intrinsically unsteady, it is shown that there are also important shortcomings when one wants to use them for stability analysis of DSWs. (C) 2005 American Institute of Physics. [DOI: 10.1063/1.1929567]
\end{abstract}

There is a class of solitary waves which appear in shallow water wave hydrodynamics, and they arise only when currents and waves interact. They are called dark solitary waves because of their similarity with similar waves in optics. The paper analyzes the structure of the bifurcation of these waves, and draws comparisons between the appearance of these waves in model equations, and their appearance in the full equations of hydrodynamics.

\section{INTRODUCTION}

The defocusing nonlinear Schrödinger (NLS) equation

$$
\mathrm{i} A_{\tau}+a A_{\xi \xi}+\beta|A|^{2} A=0, \quad a, \beta \in \mathbb{R}, \quad a \beta<0,
$$

where $\tau=\varepsilon^{2} t$ and $\xi=\varepsilon\left(x-c_{g} t\right)$ are slow time and space scales and $c_{g}$ is the group velocity, is a model for weakly nonlinear modulation of Stokes waves on the surface of a finite-depth fluid, when the dimensionless depth, $k_{0} h_{0}$, is less than the critical value $k_{0} h_{0} \approx 1.36$ (Johnson $^{11}$ ). The coefficient $a$ $=\frac{1}{2} \omega_{0}^{\prime \prime}\left(k_{0}\right)<0$ for gravity waves, where $\omega_{0}^{\prime \prime}\left(k_{0}\right)$ is the derivative of the group velocity of the linearized Stokes wave. The coefficient $\beta$ is negative (positive) for fluid depths greater (less) than the critical value. Historically, the defocusing NLS model for finite depth appears implicitly in the modulation equations of Benney and Roskes, ${ }^{3}$ and first appears explicitly in the derivation of Hasimoto and Ono. ${ }^{10}$ A review of the solutions of NLS models for water waves is given by Peregrine. ${ }^{16}$ The defocusing NLS has been observed as a model for a wide range of other physical phenomena as well (cf. Kivshar and Pelinovsky ${ }^{12}$ ).

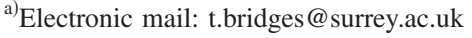

The basic periodic state, $A(\xi, \tau)=A_{0} \mathrm{e}^{\mathrm{i}(k \xi+\omega \tau)}$, of a defocusing NLS is stable, and there is a bifurcation from this periodic state to a dark solitary wave (DSW) solution. (DSWs are called "propagating phase jumps" in Ref. 10. DSWs are different from "generalized solitary waves," which also have finite oscillatory tails but the tail amplitude is much smaller than the amplitude of the core. ${ }^{14}$ ) However, all DSW solutions of (1.1) are unsteady. If an additional term is added to the NLS

$$
\mathrm{i} A_{\tau}+a A_{\xi \xi}+\beta|A|^{2} A+\mu A=0,
$$

with $\mu \neq 0$ then steady DSWs can exist. However, these waves are steady relative to a frame moving at the group velocity, since $\xi=\varepsilon\left(x-c_{g} t\right)$, and are therefore unsteady as solutions of the full water wave problem [see Eq. (1.3) for the role of $A(\xi, \tau)$ in the expression for the free-surface elevation].

Implicit in the defocusing NLS for water waves is wavegenerated meanflow. Indeed, mean-flow generated in finite depth is responsible for the change in sign of $\beta$ at the critical value of $k_{0} h_{0}$. However, there is more to mean flow than the affect on $\beta$. In the derivations of Benney and Roskes ${ }^{3}$ (hereafter BR equation) and Hasimoto and $\mathrm{Ono}^{10}$ (hereafter $\mathrm{HO}$ equation) there are one or two additional equations for mean flow. When full account of mean flow is included, one finds a range of steady and unsteady DSWs, and the connection between mean flow and the generation of DSWs can be clarified.

The purpose of this paper is to analyze the different classes of DSWs that appear in modulation equations for Stokes water waves in finite depth, with particular attention to the role of meanflow. This paper is motivated by the recent 
work of Bridges and Donaldson ${ }^{5}$ where it was shown that "secondary criticality" of Stokes traveling waves always leads to a secondary bifurcation of steady (relative to a fixed frame) DSWs of the full gravity water wave problem: These steady DSWs are pervasive at low amplitude when the depth is sufficiently small, and they are always synchronized with the Stokes waves. The bifurcation of these steady DSWs occurs when the basic Stokes wave is critical.

Attention is restricted to water waves with constant density and gravity forces only. When surface tension or density variation is present, one can find an even wider range of steady DSWs, and there is a different class of DSWs associated with bifurcations near the singularity $c_{p}=c_{g}$, where $c_{p}$ is the phase velocity. 4,9

The observation in Ref. 5 is that Stokes waves (or in general, steady periodic solutions of a Hamiltonian system) can be characterized as a three-parameter family of relative equilibria, and when a branch of such relative equilibria is degenerate it signals criticality and a homoclinic bifurcation (which represents a solitary wave). When the relative equilibrium is associated with a periodic solution, the homoclinic bifurcation results in the familiar DSW, although more general homoclinic orbits (hence solitary waves) are possible. It is essential to recognize all three parameters: In the modulation equation setting, three coupled equations are required. Neglecting one equation (i.e., using $\mathrm{HO}$ rather than BR) leads to a different (incorrect) result.

The coupled HO equations are

$$
\begin{aligned}
& \frac{\partial^{2} \varphi}{\partial T^{2}}-g h_{0} \frac{\partial^{2} \varphi}{\partial X}=\frac{g}{k_{0} \sigma}\left[2 \omega_{0} k_{0}+\left(1-\sigma^{2}\right) k_{0}^{2} c_{g}\right]\left(|A|^{2}\right)_{\xi} \\
& \mathrm{i} \frac{\partial A}{\partial \tau}+a \frac{\partial^{2} A}{\partial \xi^{2}}+\nu|A|^{2} A=\left(k_{0} \frac{\partial \varphi}{\partial X}-\left(1-\sigma^{2}\right) \frac{k_{0}^{2}}{2 \omega_{0}} \frac{\partial \varphi}{\partial T}\right) A,
\end{aligned}
$$

where $\sigma=\tanh \left(k_{0} h_{0}\right), c_{g}$ is the group velocity, $T=\varepsilon t$ and $X$ $=\varepsilon x$, and

$$
\nu=\frac{1}{2} \frac{\omega_{0} k_{0}^{2}}{\sigma^{2}}\left[\left(1-\sigma^{2}\right)^{2}-\frac{1}{2 \sigma^{2}}\left(9-10 \sigma^{2}+9 \sigma^{4}\right)\right] .
$$

The function $\varphi$ represents the modulation of meanflow, and $A$ represents the modulation of the wave. The position of the free surface for water waves, $\eta$, is related to $A$ by

$$
\eta=\varepsilon \operatorname{Re}\left[A(\xi, \tau) \mathrm{e}^{\mathrm{i}\left(k_{0} x-\omega_{0} t\right)}\right] .
$$

Neglecting the free-wave solutions of the mean flow (i.e., solutions of $\left.\varphi_{T T}-g h_{0} \varphi_{X X}=0\right)$, Hasimoto and Ono take $\varphi$ to depend only on $\xi$ and $\tau$ [see the discussion in Ref. 10 in the paragraph immediately after Eq. (4.3)], resulting in

$$
\begin{aligned}
& \frac{d}{d \xi}\left(\left(g h_{0}-c_{g}^{2}\right) \varphi_{\xi}+\frac{g}{k_{0} \sigma}\left[2 \omega_{0} k_{0}+\left(1-\sigma^{2}\right) k_{0}^{2} c_{g}\right]|A|^{2}\right) \\
& \quad=0 \Rightarrow \varphi_{\xi}=C_{0}+C_{1}|A|^{2},
\end{aligned}
$$

where $C_{0}$ is a function of integration (and to leading order a constant of integration). Setting the constant $C_{0}$ to zero and substituting $\varphi_{\xi}$ into the second equation of (1.2) leads to the
NLS equation of the form (1.1). On the other hand, when $C_{0}$ is nonzero, the NLS has an additional term

$$
\begin{aligned}
& i \frac{\partial A}{\partial \tau}+a \frac{\partial^{2} A}{\partial \xi^{2}}+\widetilde{C} A+\widetilde{\nu}|A|^{2} A=0, \quad \text { where } \\
& \widetilde{C}=-\frac{C_{0}}{2 \omega}\left(2 k_{0} \omega_{0}-k_{0}^{2} c_{g}\left(1-\sigma^{2}\right)\right),
\end{aligned}
$$

and $\tilde{\nu}$ is $\nu$ shifted by a term proportional to $C_{1}$. With $\widetilde{C}$ nonzero, steady DSWs can exist. These steady DSWs are steady relative to a moving frame, however, and are unsteady relative to a fixed frame of reference.

The steady part of (1.5) has DSWs whenever

$$
a \tilde{C}>0 \text { and } a \tilde{\nu}<0 .
$$

For gravity water waves, $a=\frac{1}{2} \omega_{0}^{\prime \prime}\left(k_{0}\right)<0$ hence steady DSWs exist whenever $\tilde{\nu}>0$ and $\widetilde{C}<0$. Since $C_{0}$ is a free parameter, steady DSWs of the HO equations are plentiful.

The derivation of the HO equations has been generalized by Branch-Nielsen and Jonsson ${ }^{8}$ and Sedletsky ${ }^{17}$ to the next order, leading to a fourth-order Dysthe-type NLS equation for finite depth. At leading order these generalizations result in two equations, a modified NLS equation plus a mean flow equation, and they agree with the $\mathrm{HO}$ equations.

However, one shortcoming of the $\mathrm{HO}$ equations, Branch-Nielsen and Jonsson's equations and Sedletsky's equations is that both components of the mean flow-mean depth and mean velocity - are determined by one equation. The modulation of mean depth is represented by $\partial \varphi / \partial T$ and the modulation of mean velocity is represented by $\partial \varphi / \partial X$.

In the earlier work of Benney and Roskes, ${ }^{3}$ independent equations are derived for modulation of mean depth and mean velocity. The BR equations are

$$
\begin{aligned}
& \frac{\partial a_{0}}{\partial T}+g b_{0}+\frac{\omega_{0}^{2}}{\sigma^{2}}\left(1-\sigma^{2}\right)|A|^{2}=0 \\
& \frac{\partial b_{0}}{\partial T}+h_{0} \frac{\partial^{2} a_{0}}{\partial X^{2}}+\frac{2 g k_{0}}{\omega_{0}} \frac{\partial}{\partial X}|A|^{2}=0 \\
& \mathrm{i} \frac{\partial A}{\partial T}+\mathrm{i} c_{g} \frac{\partial A}{\partial X}+\varepsilon\left[\frac{1}{2 \omega_{0}} \frac{\partial^{2} A}{\partial T^{2}}-\frac{g h_{0}}{2 \omega_{0}}\left(\frac{2 \omega_{0}}{g} \sigma c_{g}-1\right) \frac{\partial^{2} A}{\partial X^{2}}\right. \\
& \left.\quad+\frac{c_{g}}{\omega_{0}} \frac{\partial^{2} A}{\partial T \partial X}\right] \\
& \quad=-\varepsilon \beta|A|^{2} A+\varepsilon\left(k_{0} \frac{\partial a_{0}}{\partial X}+\frac{\omega_{0}^{3}}{2 g \sigma^{2}}\left(1-\sigma^{2}\right) b_{0}\right) A,
\end{aligned}
$$

where $a_{0}$ represents modulation of mean velocity. (To be precise it is $\partial a_{0} / \partial X$ which represents modulation of mean velocity) and $b_{0}$ represents modulation of mean depth and the coefficient of the cubic nonlinearity is 


$$
\beta=-\frac{\omega_{0} k_{0}^{2}}{4 \sigma^{4}}\left(9-10 \sigma^{2}+9 \sigma^{4}\right)=\nu-\frac{1}{2} \omega_{0} k_{0}^{2} \frac{1}{\sigma^{2}}\left(1-\sigma^{2}\right)^{2} .
$$

The first equation in (1.6) is related to Bernoulli's equation, and the second equation is related to mass conservation.

The BR equations appear to be quite different from the HO equations. For example, $\xi$ does not appear, $\varepsilon$ appears explicitly, and higher order time derivatives appear. They become closer to the $\mathrm{HO}$ equations when shifted to a frame moving with the group velocity using $\xi$,

$$
\begin{aligned}
& \frac{\partial a_{0}}{\partial T}-c_{g} \frac{\partial a_{0}}{\partial \xi}+g b_{0}+\frac{\omega_{0}^{2}}{\sigma^{2}}\left(1-\sigma^{2}\right)|A|^{2}=0 \\
& \frac{\partial b_{0}}{\partial T}-c_{g} \frac{\partial b_{0}}{\partial \xi}+h_{0} \frac{\partial^{2} a_{0}}{\partial \xi^{2}}+\frac{2 g k_{0}}{\omega_{0}} \frac{\partial}{\partial \xi}|A|^{2}=0 \\
& \mathrm{i} \frac{\partial A}{\partial \tau}+a \frac{\partial^{2} A}{\partial \xi^{2}}+\beta|A|^{2} A=\left(k_{0} \frac{\partial a_{0}}{\partial \xi}+\frac{\omega_{0}^{3}}{2 g \sigma^{2}}\left(1-\sigma^{2}\right) b_{0}\right) A
\end{aligned}
$$

neglecting terms of higher order in $\varepsilon$. These equations can be reduced to the $\mathrm{HO}$ equations by eliminating $b_{0}$ using the first equation and substituting it into the second and third equation. However, it is the full triple of equations, which separate the two contributions to the meanflow which is the most general and shows the full structure of the wave-meanflow interaction.

The BR equations are also valid for water waves with vorticity. ${ }^{6}$ To this order, vorticity has negligible effect on the wave or mean flow modulation.

The wave-meanflow interaction includes a periodic solution (due to wave modulation) coupled to drift along two directions of meanflow. A model for this is a Hamiltonian system with a three-dimensional symmetry group $S^{1} \times \mathbb{R}^{2}$, and the steady BR equations have precisely this symmetry group and the associated Hamiltonian structure.

Based on the steady BR equations, the following model is proposed for the steady coupled wave-meanflow system:

$$
\begin{aligned}
& a A_{x x}+2 \mathrm{i} b A_{x}+\beta|A|^{2} A=-2\left(\ell h_{x}+m u_{x}\right) A+\varepsilon f_{1}(A, \bar{A}, h, u) \\
& r h_{x x}+c u_{x x}=\ell\left(|A|^{2}\right)_{x}+\varepsilon f_{2}(A, \bar{A}, h, u) \\
& c h_{x x}+s u_{x x}=m\left(|A|^{2}\right)_{x}+\varepsilon f_{3}(A, \bar{A}, h, u),
\end{aligned}
$$

where $a, b, \beta, \ell, m, r, s$, and $c$ are given (in general nonzero) real parameters with $r s-c^{2} \neq 0$, and the functions $f_{j}, j=1,2,3$ are symmetry breaking functions.

When $\varepsilon=0$, this system is a generalization of the steady BR equations which are obtained by letting $a_{0}=u$ and $b_{0}$ $=h_{\xi}$ and by differentiating the first equation in (1.8). Note that $h$ is a potential for the modulation of mean depth, in the same way the $u$ is a potential for the modulation of mean velocity. (See Ref. 5 for a discussion of the role of potentials for elevation functions.) The steady BR system is recovered by taking $\varepsilon=0$ and

$$
\begin{aligned}
& b=0, \quad \ell=-\frac{\omega_{0}^{2}}{4 g \sigma^{2}}\left(1-\sigma^{2}\right), \quad m=-\frac{1}{2} k_{0}, \\
& c=-\frac{\omega_{0}}{4 g} c_{g}, \quad r=\frac{1}{4} \omega_{0}, \quad s=\frac{\omega_{0} h_{0}}{4 g},
\end{aligned}
$$

and so $r s-c^{2} \neq 0$ is equivalent to $g h_{0}-c_{g}^{2} \neq 0$.

The model Eq. (1.9) is an eighth order system of nonlinear ordinary differential equations, and it is conjectured that it is the general normal form for steady wave-meanflow coupling between a periodic wave and a two-component meanflow.

When $\varepsilon=0$ it is completely integrable. In this case, it can be explicitly solved by integrating the second and third equations

$$
r h_{x}+c u_{x}-\ell|A|^{2}=R \quad \text { and } \quad c h_{x}+s u_{x}-m|A|^{2}=Q,
$$

where $R$ and $Q$ are arbitrary constants (related to the Bernoulli constant and the mass flux, respectively). Substituting these expressions for $h_{x}$ and $u_{x}$ into the first equation of (1.9) leads to a steady NLS equation

$$
a A_{x x}+2 \mathrm{i} b A_{x}+\widetilde{\beta}|A|^{2} A+\mu A=0
$$

with

$$
\begin{aligned}
\mu= & -\frac{2}{\delta} \operatorname{det}\left[\begin{array}{lll}
r & c & R \\
c & s & Q \\
\ell & m & 0
\end{array}\right] \text { and } \tilde{\beta}=\beta \\
& -\frac{2}{\delta} \operatorname{det}\left[\begin{array}{ccc}
r & c & \ell \\
c & s & m \\
\ell & m & 0
\end{array}\right], \quad \delta=r s-c^{2} .
\end{aligned}
$$

The reduced model (1.10) has an explicit multiparameter family of DSWs

$$
\begin{aligned}
& A(x)=\mathrm{e}^{\mathrm{i} k x} s\left(k+\frac{b}{a}+\mathrm{i} \gamma \tanh (\gamma x)\right), \quad s^{2}=\frac{2 a^{2}}{|a \widetilde{\beta}|}, \\
& \gamma^{2}=\frac{1}{2 a^{2}}\left(a \mu+b^{2}-3(b+a k)^{2}\right) .
\end{aligned}
$$

However, these DSWs are interesting precisely because of their dependence on mean flow, and the explicit calculations fail to illuminate the role of mean flow.

These DSWs arise due to criticality of the coupled wave-meanflow, and criticality can be characterized in terms of quantities that can be associated with the physical phenomena. This observation, and the fact that the basic coupled wave-meanflow states arise due to symmetry and the Hamiltonian structure of the equations, suggests an organizing principle for the role of mean flow in the bifurcation of DSWs.

The steady DSW solutions which exist in the steady BR equations and the unperturbed model Eq. (1.9) arise due to the three properties of these systems: (a) They are Hamiltonian ODEs, (b) there are three symmetries: One associated with the periodic wave, and the other two associated with the fact that an arbitrary constant can be added to either $h$ or $u$; 
(c) the coupled wave-meanflow solution is a relative equilibrium. With these three properties, degeneracy of the relative equilibrium signals criticality and the bifurcation of DSWs.

An outline of the paper is as follows. The Hamiltonian structure of (1.9) is introduced in Sec. II. In Sec. III the criticality of the coupled wave-meanflow system is analyzed. In Sec. IV, the various steady and unsteady DSWs are summarized, and aspects of time modulation are discussed.

\section{HAMILTONIAN STRUCTURE OF THE MODEL EQUATION}

The Hamiltonian formulation of the system (1.9) when $\varepsilon=0$ can be obtained by first writing down the Lagrangian formulation and taking a Legendre transform. Introduce real coordinates,

$$
A=q_{1}+\mathrm{i} q_{2}, \quad h=q_{3} \quad \text { and } \quad u=q_{4}, \quad \mathbf{q}=\left(q_{1}, q_{2}, q_{3}, q_{4}\right),
$$

then the real form of (1.9) is generated by the Lagrangian

$$
\begin{aligned}
\mathcal{L}\left(\mathbf{q}, \mathbf{q}_{x}\right)= & \frac{1}{2} a\left(\left(\frac{d q_{1}}{d x}\right)^{2}+\left(\frac{d q_{2}}{d x}\right)^{2}\right)-b\left(q_{2} \frac{d q_{1}}{d x}-q_{1} \frac{d q_{2}}{d x}\right) \\
& -\left(\ell \frac{d q_{3}}{d x}+m \frac{d q_{4}}{d x}\right)\left(q_{1}^{2}+q_{2}^{2}\right) \frac{1}{2} r\left(\frac{d q_{3}}{d x}\right)^{2} \\
& +c\left(\frac{d q_{3}}{d x}\right)\left(\frac{d q_{4}}{d x}\right)+\frac{1}{2} s\left(\frac{d q_{4}}{d x}\right)^{2}-\frac{1}{4} \beta\left(q_{1}^{2}+q_{2}^{2}\right)^{2} .
\end{aligned}
$$

By taking the Legendre transform, momenta $\mathbf{p}$ $=\left(p_{1}, p_{2}, p_{3}, p_{4}\right)$ are obtained with

$$
\begin{aligned}
& p_{1}=a \frac{d q_{1}}{d x}-b q_{2}, \\
& p_{2}=a \frac{d q_{2}}{d x}+b q_{1} \\
& p_{3}=r \frac{d q_{3}}{d x}+c \frac{d q_{4}}{d x}-\ell\left(q_{1}^{2}+q_{2}^{2}\right), \\
& p_{4}=s \frac{d q_{4}}{d x}+c \frac{d q_{3}}{d x}-m\left(q_{1}^{2}+q_{2}^{2}\right) .
\end{aligned}
$$

Hence if $s r-c^{2} \neq 0$, the derivatives of $q_{j}, j=1, \ldots, 4$ can be expressed in terms of $\mathbf{q}$ and $\mathbf{p}$. Defining $H$ in the usual way, $H=\mathbf{p} \cdot \mathbf{q}_{x}-\mathcal{L}$, we find

$$
\begin{aligned}
H= & \frac{1}{2 a}\left(p_{1}^{2}+p_{2}^{2}\right)+\frac{s}{2 \delta} p_{3}^{2}-\frac{c}{\delta} p_{3} p_{4}+\frac{r}{2 \delta} p_{4}^{2}+\frac{b}{a}\left(p_{1} q_{2}\right. \\
& \left.-p_{2} q_{1}\right)+\frac{b^{2}}{2 a}\left(q_{1}^{2}+q_{2}^{2}\right)+\frac{1}{4} \tilde{\beta}\left(q_{1}^{2}+q_{2}^{2}\right)^{2}+\frac{1}{\delta}[(s \ell \\
& \left.-c m) p_{3}+(r m-c \ell) p_{4}\right]\left(q_{1}^{2}+q_{2}^{2}\right),
\end{aligned}
$$

where $\delta$ and $\widetilde{\beta}$ are defined in (1.11). The system (1.9) is then equivalent to the Hamiltonian ordinary differential equation (ODEs) $\mathbf{J} \mathbf{u}_{x}=\nabla H(\mathbf{u})$ with $\mathbf{u}=(\mathbf{q}, \mathbf{p}) \in \mathbb{R}^{8}$ and $\mathbf{J}$ the standard symplectic operator on $\mathbb{R}^{8}$. Written out, the equations are

$$
\begin{aligned}
\dot{q}_{1}= & \frac{1}{a} p_{1}+\frac{b}{a} q_{2}, \quad \dot{q}_{2}=\frac{1}{a} p_{2}-\frac{b}{a} q_{1}, \\
\dot{q}_{3}= & \frac{s}{\delta} p_{3}-\frac{c}{\delta} p_{4}+\frac{(s \ell-c m)}{\delta}\left(q_{1}^{2}+q_{2}^{2}\right), \quad \dot{q}_{4}=-\frac{c}{\delta} p_{3}+\frac{r}{\delta} p_{4} \\
& +\frac{(-c \ell+r m)}{\delta}\left(q_{1}^{2}+q_{2}^{2}\right)
\end{aligned}
$$$$
\dot{p}_{1}=\frac{b}{a} p_{2}-\frac{b^{2}}{a} q_{1}-\tilde{\beta}\left(q_{1}^{2}+q_{2}^{2}\right) q_{1}-2\left[\frac{(s \ell-c m)}{\delta} p_{3}\right.
$$$$
\left.+\frac{(-c \ell+r m)}{\delta} p_{4}\right] q_{1}
$$$$
\dot{p}_{2}=-\frac{b}{a} p_{1}-\frac{b^{2}}{a} q_{2}-\widetilde{\beta}\left(q_{1}^{2}+q_{2}^{2}\right) q_{2}-2\left[\frac{(s \ell-c m)}{\delta} p_{3}\right.
$$$$
\left.+\frac{(-c \ell+r m)}{\delta} p_{4}\right] q_{2}
$$$$
\dot{p}_{3}=0, \quad \dot{p}_{4}=0 .
$$

This system has a three parameter symmetry. An arbitrary constant can be added to $q_{3}$ and $q_{4}$ without changing the equations, and there is a rotational symmetry associated with $\left(q_{1}, q_{2}\right)$ and $\left(p_{1}, p_{2}\right)$. Formally, the symmetry is characterized in terms of a group action. Let

$$
\mathbf{G}\left(\theta_{1}, \theta_{2}, \theta_{3}\right) \mathbf{U}:=\mathbf{G}_{\theta_{3}} \mathbf{U}+\theta_{1} \mathbf{e}_{3}+\theta_{2} \mathbf{e}_{4}, \quad \mathbf{U} \in \mathbb{R}^{8},
$$

where $\mathbf{e}_{j}$ is the standard unit vector with unity in the $j$ th place and zero otherwise, and

$$
\begin{array}{ll}
\mathbf{G}_{\theta}:=\operatorname{diag}\left[\mathbf{R}_{\theta}, \mathbf{I}_{2}, \mathbf{R}_{\theta}, \mathbf{I}_{2}\right], \quad \mathbf{I}_{2}=\left[\begin{array}{ll}
1 & 0 \\
0 & 1
\end{array}\right], \\
\mathbf{R}_{\theta}=\left[\begin{array}{rr}
\cos \theta & -\sin \theta \\
\sin \theta & \cos \theta
\end{array}\right] .
\end{array}
$$

The symmetry of the Hamiltonian system is then characterized by

$H\left(\mathbf{G}\left(\theta_{1}, \theta_{2}, \theta_{3}\right) \mathbf{U}\right)=H(\mathbf{U}), \quad$ for all $\quad \boldsymbol{\theta}=\left(\theta_{1}, \theta_{2}, \theta_{3}\right) \in \mathbb{R}^{3}$.

This symmetry generates a conservation law with three components:

$R(\mathbf{q}, \mathbf{p})=p_{3}, \quad Q(\mathbf{q}, \mathbf{p})=p_{4}, \quad$ and $\quad B(\mathbf{q}, \mathbf{p})=p_{2} q_{1}-p_{1} q_{2}$.

The basic periodic wave coupled to a two-component meanflow will be characterized as a relative equilibrium associated with this group.

\section{SECONDARY CRITICALITY OF THE COUPLED WAVE-MEANFLOW SYSTEM}

In the model (1.9) with $\varepsilon=0$, the basic state is a periodic state coupled to a multicomponent mean flow. In this section it is shown that the bifurcation of DSWs occurs precisely when this coupled state is degenerate as a relative equilibrium. The mathematical degeneracy of the relative equilib- 
rium is associated with the physical criticality of the flow. ${ }^{5}$

In the coupled wave-meanflow system, the periodic wave and mean quantities can be characterized as relative equilibria associated with the symmetry (2.1). Let

$$
\mathbf{U}(x):=\mathbf{G}\left(h_{0} x, u_{0} x, k x\right) \mathbf{U}_{0}, \quad \mathbf{U}_{0}:=\left(\mathbf{q}_{0}, \mathbf{p}_{0}\right) \in \mathbb{R}^{8} .
$$

Then substitution of this expression into the governing equations shows that $\mathbf{U}_{0}$ satisfies

$$
\nabla H\left(\mathbf{U}_{0}\right)=h_{0} \nabla R\left(\mathbf{U}_{0}\right)+u_{0} \nabla Q\left(\mathbf{U}_{0}\right)+k \nabla B\left(\mathbf{U}_{0}\right),
$$

which can be characterized as a constrained variational principle. The constrained variational principle is said to be nondegenerate when

$$
\operatorname{det}\left[\begin{array}{lll}
\frac{\partial R}{\partial h_{0}} & \frac{\partial R}{\partial u_{0}} & \frac{\partial R}{\partial k} \\
\frac{\partial Q}{\partial h_{0}} & \frac{\partial Q}{\partial u_{0}} & \frac{\partial Q}{\partial k} \\
\frac{\partial B}{\partial h_{0}} & \frac{\partial B}{\partial u_{0}} & \frac{\partial B}{\partial k}
\end{array}\right] \neq 0 .
$$

When this condition fails, the basic state is degenerate in the following sense. The values of the functionals $R, Q$, and $B$ are specified. If the above determinant vanishes, then the parameters $h_{0}, u_{0}$, and $k$ can no longer be uniquely determined as functions of $R, Q$, and $B$. In Ref. 5 this degeneracy is related to criticality of the flow.

$$
\mathrm{Jac}=\left[\begin{array}{lll}
\frac{\partial R}{\partial h_{0}} & \frac{\partial R}{\partial u_{0}} & \frac{\partial R}{\partial k} \\
\frac{\partial Q}{\partial h_{0}} & \frac{\partial Q}{\partial u_{0}} & \frac{\partial Q}{\partial k} \\
\frac{\partial B}{\partial h_{0}} & \frac{\partial B}{\partial u_{0}} & \frac{\partial B}{\partial k}
\end{array}\right]=\frac{1}{\beta}\left[\begin{array}{cc}
r \beta+2 \ell^{2} & c \beta+2 \ell m \\
c \beta+2 \ell m & s \beta+2 m^{2} \\
-2 \ell(b+a k) & -2 m(b+a k)
\end{array}\right.
$$

and

$$
\begin{aligned}
\operatorname{det}(\mathrm{Jac})= & -\frac{1}{\beta^{2}}\left(\delta \tilde{\beta}\left(2 a \ell h_{0}+2 a m u_{0}+b^{2}\right)-\delta(2 \beta+\widetilde{\beta})(b\right. \\
& \left.+a k)^{2}\right) .
\end{aligned}
$$

Another illuminating form of the Jacobian is

$$
\operatorname{det}(\mathrm{Jac})=\frac{\delta}{\beta^{2}}\left(\widetilde{\beta} B_{k}+(\beta-\widetilde{\beta})(b+a k)^{2}\right) .
$$

When, $\operatorname{det}(\mathrm{Jac})=0$ the basic state is critical, and a DSW bifurcates. Although it is not immediately apparent, $\operatorname{det}(\mathrm{Jac})$ $=0$ is equivalent to $\gamma^{2}=0$ in (1.12), agreeing with the bifurcation point for DSWs in the explicit solution. This latter
The variational characterization allows closer association with the physics. The functional $R$ is related to the Bernoulli constant, $Q$ is related to the mass flux, and $B$ is related to the wave action flux.

Writing out the Lagrange Eq. (3.1) results in

$$
\begin{aligned}
& p_{1}=-(b+a k) q_{2}, \\
& p_{2}=(b+a k) q_{1}, \\
& p_{3}=\ell\left(q_{1}^{2}+q_{2}^{2}\right)+\left(r h_{0}+c u_{0}\right),
\end{aligned}
$$

and

$$
p_{4}=m\left(q_{1}^{2}+q_{2}^{2}\right)+\left(c h_{0}+s u_{0}\right)
$$

with

$$
\left(q_{1}^{2}+q_{2}^{2}\right)=\frac{1}{\beta}\left(a k^{2}+2 b k-2 \ell h_{0}-2 m u_{0}\right) .
$$

To evaluate the Jacobian in (3.2), the three invariants are evaluated along the branch of relative equilibria,

$$
\begin{aligned}
& R=\left(r+\frac{2 \ell^{2}}{\beta}\right) h_{0}+\left(c+\frac{2 m \ell}{\beta}\right) u_{0}-\frac{\ell}{\beta}\left(a k^{2}+2 b k\right) \\
& Q=\left(c+\frac{2 m \ell}{\beta}\right) h_{0}+\left(s+\frac{2 m^{2}}{\beta}\right) u_{0}-\frac{m}{\beta}\left(a k^{2}+2 b k\right) \\
& B=\frac{(b+a k)}{\beta}\left(a k^{2}+2 b k-2 \ell h_{0}-2 m u_{0}\right) .
\end{aligned}
$$

A straightforward calculation then leads to the Jacobian

$$
\left.\begin{array}{c}
-2 \ell(b+a k) \\
-2 m(b+a k) \\
3(b+a k)^{2}-b^{2}-2 \ell a h_{0}-2 m a u_{0}
\end{array}\right]
$$

equivalence is demonstrated by substituting $\mu$ in (1.11) into $\gamma$ in (1.12) and substituting $R$ and $Q$ into $\mu$.

It follows from the above result and the theory of Ref. 5 that the model Eq. (1.9) has a multiparameter family of steady DSWs. This theory recovers the explicit solution, but also shows new structure of the couple wave-meanflow problem.

Note that if this theory is applied to the steady BR Eqs. (1.8) the resulting DSWs are only quasi-steady (steady as solutions of the modulation equation but not the full waterwave problem).

In the real system, when $\varepsilon \neq 0$, new bifurcations of the DSWs will arise. However, even though the system is nonintegrable in general when $\varepsilon \neq 0$, the relative equilibrium characterization will still hold as long as the three parameter 
symmetry persists. For example, this three parameter symmetry is exact for the full water wave problem. ${ }^{5}$

\section{CONCLUDING REMARKS}

The defocusing NLS, the HO equations and the BR equations clearly have steady DSWs. However, can they have DSWs that are also steady relative to the Stokes wave? To find these latter waves, it is appropriate to consider the modulation equations before shifting to a moving frame. Starting with the original BR Eqs. (1.6) set all time derivatives to zero. The resulting modulation equation, with only the slow space scale $X=\varepsilon x$, is

$$
\begin{aligned}
& g b_{0}+\frac{\omega_{0}^{2}}{\sigma^{2}}\left(1-\sigma^{2}\right)|A|^{2}=0 \\
& h_{0} \frac{\partial^{2} a_{0}}{\partial X^{2}}+\frac{2 g k_{0}}{\omega_{0}} \frac{\partial}{\partial X}|A|^{2}=0 \\
& \frac{\mathrm{i} c_{g}}{\varepsilon} \frac{\partial A}{\partial X}-\frac{g h_{0}}{2 \omega_{0}}\left(\frac{2 \omega_{0}}{g} \sigma c_{g}-1\right) \frac{\partial^{2} A}{\partial X^{2}}+\beta|A|^{2} A+\left(k_{0} \frac{\partial a_{0}}{\partial X}\right. \\
& \left.\quad+\frac{\omega_{0}^{3}}{2 g \sigma^{2}}\left(1-\sigma^{2}\right) b_{0}\right) A=0 .
\end{aligned}
$$

Note that the coefficient of $A_{X X}$ is not $\frac{1}{2} \omega^{\prime \prime}\left(k_{0}\right)$ in this case. The coefficient $\frac{1}{2} \omega^{\prime \prime}\left(k_{0}\right)$ only shows up if a frame moving at the group velocity is chosen.

The meanflow terms $a_{0}$ and $b_{0}$ can be eliminated from the first two equations of (4.1) and substituted into the third, resulting in a steady cubic Schrödinger equation. However, the appearance of $\varepsilon$ in the denominator of the coefficient of $A_{X}$ shows that there is an imbalance in the space scales. Indeed, starting from the full steady water wave problem one finds $A_{X}=0$ at first order. In other words, for purely steady waves a different modulation equation is required. The modulation equations of $\mathrm{HO}$ and $\mathrm{BR}$ and other NLS based models are instrinsically unsteady.

A similar problem arises when one considers the stability of DSWs. The unsteady DSW solutions of (1.1) are longitudinally stable $\left(\right.$ Barashenkov $\left.{ }^{1}\right)$. (They can, however, be unstable to transverse perturbations (cf. Kuznetsov and Turitysn, ${ }^{13}$ and Pelinovsky, Stepanyants and Kivshar ${ }^{15}$ ).) But steady DSWs can be unstable (Barashenkov et al., ${ }^{2}$ De Bouard ${ }^{7}$ ). When coupled to mean flow equations, the stability properties of steady DSWs are unknown. To demonstrate stability or instability in the modulation equation setting requires an appropriate time evolution equation. The problem with the BR Eqs. (1.8) is that the mean flow evolves on a time scale $\varepsilon t$ whereas the wave modulation evolves on a time scale $\varepsilon^{2} t$. Similar problems arise with the original BR Eqs. (1.6).

In summary, the existing modulation equations for water waves in finite depth have a wide range of DSWs, but not all. They do not capture the DSWs discovered in Ref. 5 that are synchronized with Stokes waves. Existing modulation equations for time-dependent wave-meanflow interaction have mixed levels of time scales, and so may not be conclusive with regard to the stability question for steady DSWs coupled to a mean flow. A new modulation equation for steady DSWs, which includes consistent time dependence for both wave and meanflow is required.

${ }^{1}$ I. V. Barashenkov, "Stability criterion for dark solitons," Phys. Rev. Lett. 77, 1193-1197 (1996).

${ }^{2}$ I. V. Barashenkov, A. D. Gocheva, V. G. Makhankov and I. V. Puzynin, "Stability of the soliton-like bubbles," Physica D 34, 240-254 (1989).

${ }^{3}$ D. J. Benney and G. J. Roskes, "Wave instabilities," Stud. Appl. Math. 48, 377-385 (1969).

${ }^{4}$ T. J. Bridges, P. Christodoulides, and F. Dias, "Spatial bifurcations of interfacial waves when the phase and the group velocities are nearly equal," J. Fluid Mech. 295, 121-158 (1995).

${ }^{5}$ T. J. Bridges and N. M. Donaldson, "Secondary criticality of water waves. Part I. Pervasive secondary bifurcation of steady dark solitary waves," Preprint, University of Surrey (2004).

${ }^{6}$ T. Colin, F. Dias, and J.-M. Ghidaglia, "On rotational effects in the modulations of weakly nonlinear water waves over finite depth," Eur. J. Mech. B/Fluids 14, 775-793 (1995).

${ }^{7}$ A. De Bouard, "Instability of stationary bubbles," SIAM J. Math. Anal. 26, 566-582 (1995).

${ }^{8}$ U. Brinch-Nielsen and I. G. Jonsson, "Fourth-order evolution equations and stability analysis for Stokes waves on arbitrary water depth," Wave Motion 8, 455-472 (1986).

${ }^{9}$ F. Dias and G. Iooss, "Ondes solitaires noires à l'interface entre deux fluides en présence de tension superficielle," C. R. Acad. Sci., Ser. I: Math. 319, 89-93 (1994).

${ }^{10} \mathrm{H}$. Hasimoto and H. Ono, "Nonlinear modulation of gravity waves," J. Phys. Soc. Jpn. 33, 805-811 (1972).

${ }^{11}$ R. S. Johnson, "A Modern Introduction to the Mathematical Theory of Water Waves (Cambridge University Press, Cambridge, Massachusetts, 1997).

${ }^{12}$ Yu. S. Kivshar and D. E. Pelinovsky, "Self-focusing and transverse instabilities of solitary waves," Phys. Rep. 331, 117-195 (2000).

${ }^{13}$ E. A. Kuznetsov and S. K. Turitsyn, "Instability and collapse of solitons in media with defocussing nonlinearity," Sov. Phys. JETP 67, 1583-1588 (1988).

${ }^{14} \mathrm{H}$. Michallet and F. Dias, "Numerical study of generalized interfacial solitary waves," Phys. Fluids 11, 1502-1511 (1999).

${ }^{15}$ D. E. Pelinovsky, Yu. A. Stepanyants, and Yu. S. Kivshar, "Self-focusing of plane dark solitons in nonlinear defocussing media," Phys. Rev. E 51, 5016-5026 (1995).

${ }^{16}$ D. H. Peregrine, "Water waves, nonlinear Schrödinger equations and their solutions," J. Aust. Math. Soc. Ser. B, Appl. Math. 25, 16-43 (1983).

${ }^{17}$ Yu. V. Sedletsky, "The fourth-order nonlinear Schrödinger equation for the envelope of Stokes waves on the surface of a finite-depth fluid," JETP 97, 180-193 (2003). 